\title{
Overlapping and distinct neural networks supporting novel word learning in bilinguals and monolinguals
}

\begin{tabular}{|c|c|}
\hline Journal: & Bilingualism: Language and Cognition \\
\hline Manuscript ID & BLC-18-RA--0117.R2 \\
\hline Manuscript Type: & Research Article \\
\hline $\begin{array}{r}\text { Date Submitted by the } \\
\text { Author: }\end{array}$ & $n / a$ \\
\hline Complete List of Authors: & $\begin{array}{l}\text { Bakker-Marshall, Iske; University of Oxford, Department of Clinical } \\
\text { Neurosciences, Wellcome Centre for Integrative Neuroimaging, FMRIB, } \\
\text { John Radcliffe Hospital } \\
\text { Takashima, Atsuko; Radboud University Nijmegen, Donders Institute for } \\
\text { Brain, Cognition and Behaviour; Max Planck Institute for } \\
\text { Psycholinguistics } \\
\text { Fernandez, Carla; Pennsylvania State University, Psychology and Center } \\
\text { for Language Science; Duke University, Department of Psychology and } \\
\text { Neuroscience } \\
\text { Janzen, Gabriele; Donders Institute for Brain, Behaviour, and Cognition; } \\
\text { Radboud University Nijmegen, Behavioural Science Institute } \\
\text { McQueen, James; Donders Institute for Brain, Behaviour, and Cognition, } \\
\text {; Max Planck Institute for Psycholinguistics, } \\
\text { Van Hell, Janet; Penn State University, Psychology and Center for } \\
\text { Language Science }\end{array}$ \\
\hline Content Areas: & Mental Lexicon, Neurocognitive Mechanisms, Neuroimaging \\
\hline Linguistic Areas: & Not Applicable \\
\hline Methods: & Behavioural Measurements, Functional and Structural Neuroimaging \\
\hline Populations: & Adult Second Language Development, Healthy Normal Subjects \\
\hline Languages: & English, Swahili, Dutch \\
\hline Abstract: & $\begin{array}{l}\text { This study investigated how bilingual experience alters neural } \\
\text { mechanisms supporting novel word learning. English monolinguals and } \\
\text { Spanish-English bilinguals were trained on two sets of written Swahili- } \\
\text { English word pairs, one set on each of two consecutive days, and } \\
\text { performed a recognition task in the MRI-scanner. Lexical integration was } \\
\text { measured through visual primed lexical decision. Surprisingly, no group } \\
\text { difference emerged in explicit word memory, and priming occurred only } \\
\text { in the monolingual group. This difference in lexical integration may } \\
\text { indicate an increased need for slow neocortical interleaving of old and } \\
\text { new information in the denser bilingual lexicon. The fMRI data were } \\
\text { consistent with increased use of cognitive control networks in } \\
\text { monolinguals and of articulatory motor processes in bilinguals, providing } \\
\text { further evidence for experience-induced neural changes: monolinguals } \\
\text { and bilinguals reached largely comparable behavioural performance } \\
\text { levels in novel word learning, but did so by recruiting partially } \\
\text { overlapping but non-identical neural systems to acquire novel words. }\end{array}$ \\
\hline
\end{tabular}




\section{SCHOLARONE $^{\text {TH }}$ Manuscripts}


Short title: Word learning in bilinguals and monolinguals

Overlapping and distinct neural networks supporting novel word learning in bilinguals and monolinguals

Iske Bakker-Marshall ${ }^{\mathrm{a}}$, Atsuko Takashima ${ }^{\mathrm{b}, \mathrm{d}}$, Carla B. Fernandez ${ }^{\mathrm{c}, \mathrm{f}}$, Gabriele Janzen $^{\mathrm{e}, \mathrm{b}}$, James M. McQueen ${ }^{\text {b,d }}$, Janet G. van Hellc

${ }^{a}$ University of Oxford, Department of Clinical Neurosciences, Wellcome Centre for Integrative Neuroimaging, FMRIB, John Radcliffe Hospital, Headley Way, Oxford, OX3 9DU, UK

${ }^{\mathrm{b}}$ Radboud University Nijmegen, Donders Institute for Brain, Cognition and Behaviour, P.O. Box 9101, 6500 HB Nijmegen, The Netherlands

c Pennsylvania State University, Department of Psychology, University Park, PA 16802, USA

d Max Planck Institute for Psycholinguistics, Nijmegen, P.O. Box 310, 6500 AH Nijmegen, The Netherlands

${ }^{e}$ Radboud University Nijmegen, Behavioural Science Institute, P.O. Box 9104, 6500 HE Nijmegen, The Netherlands

${ }^{\mathrm{f}}$ Duke University, Department of Psychology and Neuroscience, 417 Chapel Drive, Durham, NC 27708, USA

Acknowledgements:

This research was funded by The Netherlands Organization for Scientific Research (NWO) Brain and Cognition Grant No. 433-09-239. The authors are grateful to Sarah Fairchild, Marlijn Beek and Desirae Scott for help with data collection. We would also like to thank the Penn State Social, Life, and Engineering Sciences Imaging Center, 3T MRI facility for the use of in-kind hours for data collection.

Address for correspondence:

Janet G. van Hell

Pennsylvania State University

Department of Psychology

414 Moore Building

University Park, PA 16802

USA

E-mail:jgv3@psu.edu 


\begin{abstract}
This study investigated how bilingual experience alters neural mechanisms supporting novel word learning. We hypothesised that novel words elicit increased semantic activation in the larger bilingual lexicon, potentially stimulating stronger memory integration than in monolinguals. English monolinguals and Spanish-English bilinguals were trained on two sets of written Swahili-English word pairs, one set on each of two consecutive days, and performed a recognition task in the MRI-scanner. Lexical integration was measured through visual primed lexical decision. Surprisingly, no group difference emerged in explicit word memory, and priming occurred only in the monolingual group. This difference in lexical integration may indicate an increased need for slow neocortical interleaving of old and new information in the denser bilingual lexicon. The fMRI data were consistent with increased use of cognitive control networks in monolinguals and of articulatory motor processes in bilinguals, providing further evidence for experience-induced neural changes: monolinguals and bilinguals reached largely comparable behavioural performance levels in novel word learning, but did so by recruiting partially overlapping but non-identical neural systems to acquire novel words.
\end{abstract}

Keywords: bilingualism, word learning, lexical integration, fMRI 


\section{Introduction}

Many multilinguals share the experience that with each language they master, learning the next one seems to become easier. Support for this intuition comes from classroom studies showing more successful third language acquisition in bilingual versus monolingual schools (Cenoz \& Valencia, 1994; Sanz, 2000) as well as laboratory studies using behavioural word-learning paradigms (for a review, see Hirosh \& Degani, 2018). Many of these experiments have revealed enhanced performance of bilinguals in memorising associations between novel words and translations or pictures (e.g., Kaushanskaya \& Marian, 2009a; 2009b; Kaushanskya, 2012; Papagno \& Vallar, 1995; Van Hell \& Candia Mahn, 1997; Yoshida, Tran, Benitez, \& Kuwabara, 2011), although not all studies reported better learning for bilinguals across different performance measures (e.g., Bradley, King, \& Hernandez, 2013).

Moreover, neurocognitive studies have reported that bilingualism is associated with structural brain changes. For example, increased grey matter density and white matter integrity has been found in bilingual children and adults (for reviews, see Grundy, Anderson, \& Bialystok, 2017; Li, Legault, \& Litcofsky, 2014; Pliatsikas, 2020). These anatomical changes induced by bilingual experience add to the body of research showing that specific experiences, such as musical training (e.g., Herholz \& Zatorre, 2012), juggling (Draganski et al., 2004), experience with spatial navigation (e.g., Maguire et al., 2000) and action video gaming (e.g., Bavelier \& Davidson, 2013) can modify brain structure and induce long-term changes in cognitive behaviour. Functional neuroimaging studies have also studied the impact of bilingual experience on linguistic function and on non-linguistic cognitive and executive function (e.g., Bradley, King, \& Hernandez, 2013; Kovelman, Baker, \& Petitto, 2008; for a recent review, see Bialystok, 2017). The fMRI study reported in this paper examined how bilingual experience may alter neural mechanisms associated with learning 
novel foreign language words, and the extent to which these mechanisms are different for bilinguals and monolinguals. More specifically, the current study aimed to shed light on this question from a memory integration perspective, testing the hypothesis that experience with learning a second language may alter the integration process of novel lexical-semantic information with prior knowledge.

Researchers who observed enhanced performance of bilinguals on learning and memorising novel words have proposed different explanations for this effect. One explanation is based on the idea that the experience of developing two language systems leads to better developed phonological abilities. For example, bilingual children outperform their monolingual peers on measures of phonological awareness, such as phoneme substitution in a task like "take away the first sound of the word 'cat', and put in the $/ \mathrm{m} /$ sound of the word "mop'” (e.g. Bialystok, Majumder, \& Martin, 2003). This advantage may benefit learning of new phonological forms. However, the phonological awareness advantage only occurs in bilinguals whose languages share the same print-to-sound conversion principles (Bialystok et al., 2003; 2005). If phonological awareness underlies the bilingual advantage in word learning, only bilinguals whose languages share print-to-sound conversion principles should perform better than monolinguals on word learning tasks. In contradiction of this prediction, a word learning advantage has been demonstrated both in bilinguals speaking alphabetic languages (English and Spanish) and in speakers of English and Mandarin, which have different print-to-sound conversion systems (Kaushanskaya \& Marian, 2009a).

Relatedly, it has been argued that phonological memory may underlie the bilingual advantage in word learning, given that multilingual experience is correlated with higher performance on auditory digit span and non-word repetition tasks (Papagno \& Villar, 1995). In order to test this hypothesis, Kaushanskaya (2012) compared monolinguals and bilinguals 
on a digit span task. Monolinguals were divided into a low-span and high-span group. Bilinguals were matched to the high-span monolinguals (as it proved impossible to find lowspan bilinguals). High-span monolinguals performed better than low-span monolinguals on phonologically unfamiliar novel words, but the bilinguals outperformed both groups of monolinguals on both familiar and unfamiliar words. Thus, even when matched on phonological memory, bilinguals exhibited a word learning advantage over monolinguals. These findings suggest that phonological memory and awareness may be beneficial in word learning, but cannot account completely for the bilingual advantage.

A second line of research proposes that the bilingual advantage in word learning is driven by enhanced cognitive control, which reduces native-language interference (Bartolotti et al., 2011; Bartolotti \& Marian, 2012; Bradley, King, \& Hernandez, 2013; Kaushanskaya \& Marian 2009a). Because lexical items in the bilingual lexicon compete both within and across languages (e.g. Marian \& Spivey, 2003; Van Heuven, Dijkstra, \& Grainger, 1998), bilinguals have more experience in resolving competition and suppressing irrelevant information than monolinguals. This has been claimed to reduce the interference of native-language competitors during retrieval of newly learned words, which may result in facilitated access to novel words and thus better memory performance (Bartolotti \& Marian, 2012). Similarly, bilinguals have been shown to be less hindered than monolinguals by novel orthographyphonology mappings that are inconsistent with their native languages, possibly because of their experience with inhibiting irrelevant rules (Kaushanskaya \& Marian, 2009b). In line with this view, Bradley et al. (2013) found that bilinguals engaged a less extensive network of brain regions implicated in cognitive control than monolinguals during retrieval of newly learned words, which they suggested reflects more efficient and automatic access to novel representations in bilinguals. More specifically, between-groups comparisons revealed that the monolingual word learners showed activation of the right dorsolateral prefrontal cortex 
(DLPFC), right supplementary motor area (SMA), left anterior cingulate cortex (ACC), and the left caudate in response to newly learned words, whereas the bilinguals showed activation of the left putamen in response to newly learned words. Increased activation of the left putamen for a non-native language in multilinguals, as well as increased grey matter density in the left putamen of multilinguals relative to monolinguals, has also been found by Abutalebi et al. (2013). The DLPFC, SMA, ACC, caudate, and putamen are part of the language control network proposed by Abutalebi and Green $(2007 ; 2016)$, the Adaptive Control Hypothesis, in which the left putamen is typically associated with motor processes and control of articulatory processes (for a comparable model emphasizing frontal-striatal pathways, see Stocco, Yamasaki, Natalenko, \& Pratt (2014). Accordingly, Bradley et al. (2013) proposed that in novel word learning, monolinguals rely on a higher-level mode of cognitive control by recruiting a set of prefrontal cognitive control regions and the caudate, whereas bilinguals show more localised activity within the subcortical striatum, in particular the putamen, which is associated with more direct motor control of language.

A third approach to explain word learning benefits in bilinguals focuses on the semantic nature of the bilingual lexicon and on the way novel words are encoded. Studying how semantics affects word learning, Kaushanskaya and Rechtzigel (2012) taught monolinguals and early bilinguals translations of concrete words such as 'daisy', and abstract words such as 'virtue'. They found that concrete words were better remembered than abstract words, and this concreteness effect was larger in bilinguals than in monolinguals.

Concreteness effects are generally interpreted to reflect richer semantic processing of concrete words, for instance because storage of concrete words involves both verbal and perceptual information (Paivio, Walsh, \& Bons, 1994) or because their representations contain more conceptual information than abstract words (De Groot, 1989). Kaushanskaya and Rechtzigel (2012) argued that their observed larger concreteness effect in bilinguals may 
reflect increased semantic activation elicited by concrete words in the bilingual lexicon, as concrete words have more similar semantic representations across languages (De Groot, 1992) ${ }^{1}$. The richer semantic network associated with concrete words in bilinguals as compared to monolinguals, and higher levels of semantic activation in the bilinguals' versus monolinguals' lexical-semantic network, may thus benefit the formation of connections between novel and existing words in bilinguals, leading to enhanced memory for novel words with concrete referents (as used in the present study).

This possibility resonates with the idea that the integration of novel words into the existing mental lexicon is crucial to their lexical functioning (Davis \& Gaskell, 2009; Gaskell \& Dumay, 2003; Leach \& Samuel, 2007). This integration process is thought to involve a representational shift from the hippocampal system to a widely distributed neocortical network (Davis \& Gaskell, 2009; Frankland \& Bontempi, 2005; Marr, 1970; McClelland, McNaughton, \& O’Reilly, 1995; Squire \& Alvarez, 1995; Takashima, Bakker, Van Hell, Janzen, \& McQueen, 2014; Takashima, Marshall-Bakker, Van Hell, McQueen, \& Janzen, 2019). This neocortical network entails posterior and anterior temporal regions (with left hemisphere bias) that overlap with the ventral speech processing pathway proposed by Hickok and Poeppel (2007), including the posterior and anterior middle temporal gyri (pMTG and aMTG) and posterior and anterior inferior temporal sulci (pITS and aITS), as well as regions overlapping with the (left-dominant) dorsal speech processing pathway (Hickok \& Poeppel, 2007), including pars opercularis and pars triangularis of the inferior frontal gyrus (Broca's area; for more details, see Davis \& Gaskell, 2009); originally proposed for spoken words, the Davis and Gaskell's (2009) neocortical network also entails regions involved in phonological processing, including the superior temporal gyrus (STG). In this view, the hippocampus rapidly binds the components of a memory trace during encoding, and is essential for immediate retrieval. In the hours, days or even weeks after the learning event, 
a gradual process of integrating the new information into the existing neocortical memory network unfolds. Ultimately, the novel representation becomes completely neocortically represented and no longer engages the hippocampus during retrieval. As information in the hippocampus decays more rapidly than cortico-cortical connections, this offline process consolidates the novel memory and protects it from forgetting.

The integration of a novel word with existing neighbouring form- and meaningrelated words allows it to interact with those neighbours during language processing. For instance, novel words that overlap phonologically with existing words (e.g. 'cathedruke' overlaps with 'cathedral') slow down recognition of those neighbours after a consolidation period of several hours or days, but not immediately after training (e.g. Bakker, Takashima, Janzen, Van Hell, \& McQueen, 2014; Dumay \& Gaskell, 2007; 2012; Gaskell \& Dumay, 2003). Secondly, meaningful novel words start to facilitate recognition of semantically related existing words after a consolidation period (Tamminen \& Gaskell, 2013; Van der Ven, Takashima, Segers, \& Verhoeven, 2015). The emergence of lexical-semantic integration effects has been proposed to rely on the formation of a lexical representation in the posterior middle temporal gyrus (pMTG) (Bakker-Marshall, Takashima, Janzen, Van Hell, \& McQueen, 2018; Takashima et al., 2014). Many current models of word processing view the pMTG as a lexical hub that mediates the mapping of word forms onto distributed semantic information (Gow, 2012; Hickok \& Poeppel, 2004; 2007; Lau, Phillips, \& Poeppel, 2008).

Recent findings suggest that encoding factors can influence the success and pace of this lexicalisation trajectory. For instance, interleaved exposure to novel words and their existing phonological neighbours during training accelerates the emergence of competition (Lindsay \& Gaskell, 2013), suggesting that activation of the existing lexical-semantic network during encoding stimulates the integration of novel words. These observations are in line with rodent studies showing that when novel information can be related to a coherent 
network of prior knowledge, newly formed representations can rapidly become neocortically integrated and independent of the hippocampus (Tse et al., 2007).

An interesting possibility therefore is that the enhanced lexical-semantic activation in the bilingual lexicon during processing of a novel word suggested by Kaushanskaya and Rechtzigel (2012) leads to faster and more successful lexicalisation, giving the increased opportunity for integration by formation of neocortical connections. This hypothesis leads to several specific predictions. Firstly, bilinguals should exhibit the largest word learning benefit relative to monolinguals shortly after training, as monolinguals may reach the same level of lexicalisation after an opportunity for offline consolidation. This prediction is borne out by a number of studies showing a bilingual advantage at immediate test but smaller or no effects after a week (Kaushanskaya \& Marian, 2009a; 2009b; but cf. Kaushanskaya, 2012; Van Hell \& Candia Mahn, 1997). Secondly, bilinguals may be expected to show larger semantic priming effects between novel and existing words than monolinguals, and priming effects may require less of a delay in bilinguals than in monolinguals. Thirdly, retrieval of recently learned novel words should be supported by a more neocortical lexical-semantic network (as outlined above) in bilinguals as compared to monolinguals.

The current study aimed to put these predictions to the test. A group of English monolinguals and a group of early Spanish-English bilinguals learned two sets of SwahiliEnglish translation pairs on two consecutive days, followed by a testing session on the second day. This design made it possible to contrast a recent condition (words learned just before testing on the second day) with a remote condition (words learned on the first day) within the same testing session. We used a semantic priming task to probe immediate and delayed lexical-semantic integration effects. Furthermore, blood-oxygen-level-dependent (BOLD) responses during word retrieval were measured to examine group differences in the contribution of neocortical areas involved in lexical-semantic processing to the retrieval of 
recent and remote words. Additionally, we investigated whether, as suggested by Bradley et al. (2013), monolinguals rely more on cognitive control areas while recognising learned words (showing enhanced activation in the DLPFC, SMA, ACC, and caudate nucleus) relative to bilinguals, and whether this pattern changes with consolidation.

We furthermore tested whether the monolingual and bilingual groups differed on nonlinguistic cognitive control by means of a Flanker task (Eriksen \& Eriksen, 1974). Bilinguals have often been reported to outperform monolinguals on tasks requiring inhibition and attention switching (e.g., Costa, Hernández \& Sebastián-Galles, 2008; for review see Bialystok, Craik \& Luk, 2012), although it has been argued that this bilingual advantage is less robust in young adults (Bialystok, 2017), or may only be observed under restricted circumstances (e.g., Hilchey \& Klein, 2011; Lehtonen et al., 2018; Paap, Johnson \& Sawi, 2015).

\section{Methods}

\section{Participants}

Twenty-four English monolingual participants (nine males, aged 18-24 years, mean 20) and 24 Spanish-English bilingual participants (eleven males, aged 18-34 years, mean 24) were recruited from the Penn State University community. Participants reported having no history of learning- or language-related problems, had normal or corrected-to-normal vision and hearing, and were right-handed. One monolingual participant was excluded from all analyses due to extremely poor performance on the fMRI and memory tasks. One further monolingual participant was excluded due to experimenter error.

Monolingual participants were selected to have no experience with a foreign language apart from beginner-level courses in high school or university. A total of 15 monolinguals 
reported having some experience learning a foreign language (Spanish, French, Italian or Latin). Self-rated proficiency in this language was 1.9 on average on a scale of 1 (none) to 10 (native-like), and current frequency of use was 0.25 on average on a scale of 0 (never) to 10 (constantly).

The bilingual group consisted of early, mostly sequential Spanish-English bilinguals. All bilingual participants acquired Spanish from birth, and started learning English between the ages of 0 and 10 years (mean 5). Proficiency in English was self-rated between 8 and 10 (mean 9.3), and in Spanish between 7.3 and 10 (mean 9.5), on a scale of 1 (none) to 10 (native-like). Six participants considered themselves most proficient in English, fourteen in Spanish, and four reported equal proficiency in both languages. A Boston Naming Test (BNT) was performed in English by the monolinguals and in English and Spanish by the bilinguals (see 2.2). Naming data for one monolingual and one bilingual participant was lost for the English BNT, and for one bilingual for the Spanish BNT due to technical problems. Monolinguals scored better than bilinguals on the English BNT (51 versus 42 words correct out of $60, t(42)=4.952, p<.001)$. Bilinguals did not score differently on the English and Spanish versions (42 versus 41 words correct, $t(21)=.551, p=.588)$. Analyses of their selfrated proficiency showed that bilinguals who reported that English was their most proficient language or that both languages were equally proficient scored higher on the English BNT than the bilinguals who reported being most proficient in Spanish $(t(21)=4.037, p<.001)$ and only marginally lower than the monolinguals $(t(29)=1.934, p=.063)$. These outcomes suggest that self-ratings corresponded well with objective language proficiency measures.

On a scale of 1 (never) to 10 (always), bilingual participants reported using English most when reading (9.6), speaking with friends (7.5) and at work or school (9.8). Sixteen participants used English with their families, with an average frequency of 5.5. Spanish was most commonly spoken when interacting with family (8.6), but was also used with friends 
(5.5), when reading (6.3), and at school or work (3.1). Participants estimated using English $40-90 \%$ of the time (mean $73 \%$ ) and Spanish 10-55\% (mean 26\%). All participants reported using both languages on at least one day a week (English: mean 6.6 days a week; Spanish: mean 5.0 days a week). Six participants considered their current dominant language to be English, ten Spanish, and eight considered both languages equally dominant.

Fifteen bilinguals reported having experience with an additional (third) language (French, German, Italian or Catalan). They rated their proficiency in this language as 3.4 on average on a scale of 1 (none) to 10 (native-like) and reported an average usage frequency of 1.5 on a scale of 0 (never) to 10 (constantly).

\section{Procedure}

On day 1, participants were trained on a set of 30 Swahili-English word pairs. Additionally, they performed a Flanker task and a set of language proficiency tasks and completed a language history questionnaire. On day 2, approximately 24 hours later, participants were trained on a second set of 30 Swahili-English word pairs. Immediately afterwards, they performed a recognition task in the MRI scanner. This task included the Swahili words learned the day before ('remote'), the Swahili words learned the same day ('recent'), Swahili words that were not part of the trained set ('untrained') and existing English words ('existing'). Following the scanning session, semantic integration was assessed by means of a lexical decision task in which the learned Swahili words primed semantically related or unrelated English words. Finally, participants were tested on explicit memory for the studied words with four memory tasks of varying difficulty.

\section{Language proficiency}


Monolingual participants performed the BNT, consisting of 60 pictures, in English. Blackand-white line drawings of objects were presented on the screen one by one, and participants were instructed to name each picture as quickly and as accurately as possible. Each picture was presented for 5 seconds or until the participant pressed a button. Bilingual participants performed the BNT in English first. They then did the Flanker task (see below) and subsequently performed the BNT in Spanish.

\section{Flanker task}

A version of the Flanker task (Eriksen \& Eriksen, 1974) containing a go/no-go element as developed by Luk (2008) was used as a measure of cognitive control, and was administered to the monolingual and bilingual participants. Participants pressed one of two buttons to indicate the direction a red chevron was pointing. In the control condition, only the target chevron was presented. In the congruous condition, the red chevron was flanked by four black chevrons pointing in the same direction. In the incongruous condition the four distractor chevrons pointed in the opposite direction. In the neutral condition the four distractors were diamonds, and in the no-go condition the four distractors were crosses indicating that the participant should refrain from giving a response. Conditions were presented in a blocked design consisting of seven blocks: a control block, a congruous/incongruous block, a neutral/no-go block, a mixed block containing all conditions except the control condition, a second neutral/no-go block, a second congruous/incongruous block, and a second control block. Control blocks were always presented first and last, the mixed block was always presented in the middle, and the order of the congruous/incongruous and neutral/no-go blocks was counterbalanced across participants. Trials consisted of a 500 ms fixation screen followed by stimulus presentation for $2000 \mathrm{~ms}$ or until button press. The type of trial, number of left and right responses, and target position (second, third or fourth 
symbol) was counterbalanced within each block. The order of trials within blocks was randomised per participant.

\section{Training}

The novel word training procedure comprised four 'encoding' blocks and four active tasks. In the encoding blocks a Swahili word was presented visually alongside its English translation, both in lower case letters, for four seconds. Participants were instructed to say both words out aloud once. The active tasks were 1) four-alternative forced-choice (4AFC) matching of a Swahili cue to its English translation, 2) typing the English translation in response to a Swahili word, 3) 4AFC matching of an English cue to its Swahili translation, and 4) typing of the Swahili translation in response to an English word. There was no time pressure, and the correct answer was presented for two seconds after a response was given. The four active tasks were presented in the order listed above, with an encoding block preceding every active task block. Each word was presented once in each block, giving a total of eight exposures per word.

\section{fMRI task}

The fMRI task on day 2 contained Swahili words from both days (recent and remote), untrained Swahili words, and existing English words. Each word was presented once, for a total of 120 trials, interspersed with 30 null events of four seconds. The recognition memory task was split into three runs, with each run containing an equal number of trials from each condition. The order of items within a run was randomised per subject and the order of runs was counterbalanced.

A trial started with presentation of a single word in the centre of the screen for two seconds. After a variable interstimulus interval (ISI) of one to three seconds, a question 
prompt appeared on the screen. Participants indicated whether or not the presented word was part of the set of Swahili words they learned. Responses were given by pressing one of two buttons on a button box held in the left hand. The response prompt remained on the screen for two seconds. After an ISI of one second, a second response screen appeared prompting participants to indicate whether or not they knew the meaning of the word. The response options again remained on the screen for two seconds. The next trial started after a variable inter-trial interval (ITI) of three to seven seconds.

\section{Semantic priming}

The priming task on day 2 required participants to make a lexical decision to English words and pseudo-English nonwords presented in capital letters, as fast and as accurately as possible. Each target was preceded by a learned Swahili prime word, presented in lower case letters. Each target word had one semantically related prime (e.g. farasi (horse) - SADDLE) and one unrelated prime (e.g. kijiko (spoon) - SADDLE). Each Swahili word, in turn, primed two different semantically related targets (e.g. farasi - SADDLE and DONKEY). The task thus consisted of 240 word trials (60 learned Swahili words with two unrelated and two related target words each). For the purpose of the lexical decision task, 240 pseudo-word targets were added which were also primed by the 60 learned Swahili words. Repetitions of the same prime were separated by at least 30 trials, and repetitions of the same target by at least 60 trials. Each trial started with a $500 \mathrm{~ms}$ fixation, followed by the prime for $250 \mathrm{~ms}$, a blank screen for $250 \mathrm{~ms}$, and the target for $1500 \mathrm{~ms}$ or until the participant responded. Responses were given by pressing the right index finger button for 'yes' and the left index finger button for 'no' on a button box.

Memory tasks 
A series of four tasks of decreasing difficulty measured the strength of novel word memories on day 2. In the free recall task, presented first, participants were instructed to write down as many Swahili words as they could remember, without their translation, in five minutes. The other tasks, in order of presentation, were: typing the Swahili translation of an English word (identical to training task 4), typing the English translation of a Swahili word (identical to training task 2), and matching of a Swahili word to one of four English words (4AFC task; identical to training task 1). No feedback was given in these tasks.

\section{Stimuli}

\section{Training and fMRI task}

Two lists of 30 Swahili-English word pairs were created. The allocation of lists to training sessions (recent/remote) was counterbalanced across subjects within each group (monolinguals/bilinguals). Each word referred to a common, concrete object. Swahili words were 4-6 letters long (mean 5). Although not all words obeyed English phonotactics, they were easily pronounceable for English speakers. None of the Swahili words were cognates of English or Spanish words. The 30 untrained Swahili words used in the fMRI task were selected to match the learned Swahili words in length (4-7 letters, mean 5) and orthographic features. The existing English words used in the fMRI task were 4-7 letters long (mean 5), had a CELEX (Baayen, Piepenbrock, \& Gulikers, 1995) frequency of 2-45 occurrences per million (mean 26) and were not closely semantically or orthographically related to any of the learned word pairs. The full set of stimuli is listed in Table 1.

$<$ Insert Table 1 about here $>$

\section{Priming task}


Word targets in the priming task were 3-8 letters long (mean 5) and had a CELEX frequency of 1-288 (mean 44) per million. Orthographically and phonologically legal pseudo-word targets were derived by substitution of one letter from English words that were not part of the stimulus set, and matched in length with the word targets.

\section{fMRI acquisition}

fMRI data were recorded in a Siemens Magnetom Trio 3T MRI scanner at the SLEIC center at Penn State, using a 12-channel head coil. Functional images were acquired using a $\mathrm{T} 2 *$-weighted gradient planar imaging sequence with $\mathrm{TR}=2.01 \mathrm{~s}, \mathrm{TE}=30 \mathrm{~ms}, 34$ slices, descending slice order, slice thickness $3.0 \mathrm{~mm}$, slice gap $0.5 \mathrm{~mm}$, matrix size $64 \times 64$, field of view $\left(\right.$ FOV) $224 \times 224 \mathrm{~mm}$, flip angle $90^{\circ}$, and voxel size $3.5 \times 3.5 \times 3.0 \mathrm{~mm}$. Slices were angulated in an oblique axial manner. T1-weighted anatomical scans at $1 \mathrm{~mm}$ isotropic resolution were acquired with TR $1650 \mathrm{~ms}$, TE $2.03 \mathrm{~ms}$, 160 slices, flip angle $9^{\circ}$, matrix size $256 \times 256$.

\section{fMRI analysis}

Preprocessing was performed using SPM8 (www.fil.ion.ucl.ac.uk). Functional images were first realigned and resliced to the first volume of the first run, and the mean functional image was co-registered with the subject's structural MRI using the mutual information optimization function implemented in SPM8. Images were slice-time corrected to the 17th slice using Fourier pane shift interpolation. The structural MRI was segmented and normalized to SPM8's T1 template in Montreal Neurological Institute (MNI) space. Functional images were normalized to MNI space and smoothed with an $8 \mathrm{~mm}$ full-width half maximum Gaussian filter. 
Data were modelled using a general linear model (GLM) with four experimental regressors: 1) remote Swahili words that were correctly matched to their English translation in the 4AFC memory task, 2) recent Swahili words correct in the 4AFC task, 3) untrained Swahili words, and 4) existing English words. Two other regressors were also included: recent/remote Swahili words that were incorrectly responded to in the 4AFC task ('misses'), and null events. For the two participants that scored $100 \%$ correct on the $4 \mathrm{AFC}$ task, a dummy trial of the 'misses' regressor was inserted 20 seconds after the last real trial to keep the GLM model consistent across all participants. A stick function (time-locked to the onset of word presentation) representing the explanatory variables was convolved with the canonical hemodynamic response function provided by SPM8. Six motion parameters were added as additional regressors to account for motion artefacts. Data was high-pass filtered using a 128 s cut-off to reduce the effects of low-frequency drift. Each experimental condition was contrasted against an implicit baseline on the first level. The resulting images were entered into a full-factorial second-level model with factors Group (monolingual, bilingual) and Condition (remote, recent, untrained, existing).

For whole-brain analyses, voxels were thresholded at $p=.001$ uncorrected $\left(p_{\text {unc }}\right)$ and cluster statistics were evaluated at $p=.05$ family-wise error corrected ( $p_{F W E}$, Hayasaka \& Nichols, 2003). The contrast existing $>$ untrained words with a stricter voxel threshold of $p_{F W E}=.05$ was used to define regions involved in lexical processing. The significant clusters of this contrast were used as lexicality regions of interest (ROIs) to examine the orthogonal effects of training and consolidation, and differences between the groups within the restricted areas of the brain that are responsive to lexicality. ROI analysis was performed using an initial voxel threshold of $p_{u n c}=.001$ and applying a small volume correction (SVC; $\left.p s v c\right)$ for multiple comparisons, evaluated at cluster-level at $p_{F W E}=.05$ for each of the clusters. To investigate the contribution of areas related to cognitive control, ROIs were created by 
drawing a $15 \mathrm{~mm}$ sphere around the peak voxels of the group differences reported by Bradley et al. (2013). These included the right inferior/middle frontal gyri (IFG/MFG; labelled DLPFC by Bradley et al. [42 23 16]), right SMA ([15 -1 49]), left caudate ([-15 23 10]) and left putamen/pallidum ([-27 -7 -2]). Since the peak voxel of the cluster observed in the ACC ROI by Bradley et al. was in white matter lateral to the ACC, we defined the ACC ROI anatomically based on the AAL atlas (Tzourio-Mazoyer et al., 2002).

\section{Results}

\section{Cognitive control (Flanker task)}

Errors and response times below $50 \mathrm{~ms}$ or above $1500 \mathrm{~ms}$ were excluded from the reaction time (RT) analysis (10\%). Data for one monolingual participant were lost due to experimenter error.

Overall accuracy did not differ between groups $(F(1,43)=.242, p=.625)$ and there was no Group by Condition (blocked congruent, blocked incongruent, blocked go, blocked no-go, mixed congruent, mixed incongruent, mixed go, mixed no-go, control) interaction $(F(1.1,47.7)=.01, p=.938)$.

The change in RT relative to the control condition was computed for the congruent and incongruent trials in the congruent/incongruent blocks; see Figure 1, panel A. Incongruent trials were responded to slower $(F(1,43)=132.733, p<.001)$, reflecting the basic Flanker effect. There was no effect of $\operatorname{Group}(F(1,43)=.07, p=.792)$ nor an interaction $(F(1,43)=.039, p=.845)$.

For the congruent, incongruent and neutral trials in the mixed block, the change in RT relative to the same trial type in the blocked conditions was computed; see Figure 1, panel A. This mixing cost reflects participants' response flexibility in responding when trial types are 
intermixed, and the cost of monitoring and coordinating multiple streams of information. Bilinguals exhibited smaller mixing costs than monolinguals across conditions (20 ms and 42 $\mathrm{ms}$, respectively; $F(1,43)=4.143, p=.048)$. This effect has been taken to reflect bilinguals' experience of monitoring and coordinating two simultaneously active languages (e.g., Barac \& Bialystok, 2012; Gold, Kim, Johnson, Kryscio, \& Smith, 2013; Yang, Hartanto, \& Yang, 2016; Wiseheart, Viswanathan, \& Bialystok, 2016). A main effect of Condition $(F(2,86)=$ 21.324, $p<.001)$ reflected significant overall mixing costs in the incongruent condition (57 $\mathrm{ms}, t(44)=6.503, p<.001)$ and the congruent condition $(25 \mathrm{~ms}, t(44)=4.613, p<.001)$ but not in the go condition $(8 \mathrm{~ms}, t(44)=1.179, p=.245)$. There was no interaction of Condition by $\operatorname{Group}(F(2,86)=.113, p=.893)$. See Figure 1 for an illustration of the Flanker effect and mixing costs by group.

$<$ Insert Figure 1 about here $>$

\section{Training and Memory (behavioural results)}

\section{Training}

Training data for one monolingual participant was lost due to technical problems.

Performance on the training tasks was generally good, with scores on the most difficult task (typing the Swahili word cued by its English translation) reaching over 50\% on average on both days. The English-Swahili 4AFC matching task produced the highest scores; $85 \%$ on day 1 and $87 \%$ on day 2 . The Swahili-English 4 AFC matching task was performed better on day $2(67 \%)$ than on day $1(61 \%, F(1,43)=8.7, p=.005)$. No other tasks exhibited any differences between days or groups. 


\section{fMRI old/new recognition task}

For one monolingual participant, behavioural data was not analysed because button presses were not recorded in one of the runs. An ANOVA with factors Day (remote, recent) and Group (monolinguals, bilinguals) did not reveal any effects of Day or Group on old/new recognition for the learned words. Remote words were recognised in $89 \%$ of trials, recent words in $91 \%$ of trials. A main effect of Day was found on responses to the second question ('Do you know the meaning of this word?'), with the meanings of recent words being remembered better than those of remote words $(72 \%$ versus $50 \% ; F(1,43)=76.39, p<.001)$. No effect of Group or an interaction was observed.

\section{Memory tasks}

Participants freely recalled more words from the recent (25\%) than the remote $(21 \%)$ condition $(F(1,44)=4.08, p=.049)$. Similarly, when asked to type the Swahili word in response to an English word, participants scored higher on the recent (39\% correct) than on the remote $(30 \%$ correct $)$ condition $(F(1,44)=10.32, p=.002)$. Typing the English word when cued by its Swahili translation was also easier for the recent (66\% correct) than the remote $(48 \%$ correct $)$ condition $(F(1,44)=62.78, p<.001)$, as was Swahili-English 4AFC matching (recent $90 \%$, remote $83 \%$ correct; $F(1,44)=24.41, p<.001$ ). In none of the memory tasks was an effect of Group or an interaction of Group by Condition observed (see Figure 1).

\section{Semantic priming}

Errors and responses below 200 or above $1700 \mathrm{~ms}$ were removed (10\%). Data for one bilingual participant was lost. This final data set is summarised in Figure 1 Panel C. An ANOVA with factors Day (remote, recent) x Relatedness (related, unrelated) and between- 
subjects factor Group (monolinguals, bilinguals) revealed a main effect of Day $(F(1,43)=$ $4.42, p=.041)$, reflecting overall slower responses in the remote condition. A main effect of Group indicated a small overall reaction time advantage for monolinguals $(F(1,43)=4.1, p=$ .049). No main effect of Relatedness was observed, though there was a modest trend towards an interaction of Relatedness x Day $(F(1,43)=2.57, p=.12)$. There was no three-way interaction. Planned paired t-tests of the related versus unrelated conditions within each group suggested a small priming effect in the remote condition for the monolinguals $(12 \mathrm{~ms}, t(21)=$ $2.33, p=.03)$, but not in the recent condition $(-3 \mathrm{~ms}, p=.61)$. In the bilingual group no evidence of priming was observed in the remote $(-2 \mathrm{~ms}, p=.62)$ or recent $(2 \mathrm{~ms}, p=.76)$ condition.

\section{fMRI results}

\section{Lexicality effects}

Existing English words elicited more activation than untrained Swahili words in the left middle temporal gyrus (MTG), bilateral angular gyrus (AG), left dorsal and orbital parts of the middle frontal gyrus (MFG), and bilateral precuneus with a left-lateralised peak (see Figure 2). The reverse contrast did not reveal any significant clusters. There was no interaction with Group. These areas, identified as responsive to lexicality, were used as lexicality ROIs to examine the effects of learning and consolidation and differences between the monolingual and bilingual groups in the next analyses.

$<$ Insert Figure 2 about here $>$

Learning and consolidation 
Recently learned words elicited more activation than untrained Swahili words in the left inferior parietal lobe (IPL) and AG, anterior cingulate cortex (ACC), superior frontal gyrus (SFG), dorsal and orbital parts of the MFG, pars triangularis of the inferior frontal gyrus (IFG), precuneus and posterior cingulate cortex, and bilateral caudate. The identical pattern was observed for remote versus untrained Swahili words (see Figure 3). ROI analysis of regions where a lexicality effect was observed, but which did not show learning effects (contrast of learned-untrained words) at the whole-brain level (the left MTG and right AG), revealed a learning effect in the left MTG for both recent ([-58 -40 -10], cluster-size $\mathrm{k}=52$ voxels, $\left.p_{S V C}=.012\right)$ and remote $\left([-45-38-12], \mathrm{k}=66, p_{S V C}=.01\right)$ words. There was no interaction with Group in either condition. Neither the contrasts remote-recent and recentremote nor the interactions of these contrasts with group revealed any suprathreshold voxels. No correlation was found between lexical activation and behavioural priming effects for recent or remote words.

$<$ Insert Figure 3 about here $>$

\section{Group differences}

For the contrast of all words relative to baseline, monolinguals showed greater activation than bilinguals in several of the lexicality ROIs: the left precuneus ([0 -64 44], $\mathrm{k}=25, p_{S V C}=$ $.016), \operatorname{MFG}\left([-144648], \mathrm{k}=85, p_{S V C}=.016\right)$, and MTG $\left([-62-36-8], \mathrm{k}=11, p_{S V C}=.029\right)$. Within the cognitive control-related ROI, in line with Bradley et al. (2013), monolinguals also engaged a right IFG/MFG region more ([36 30 18], $\left.\mathrm{k}=62, p_{S V C}=.018\right)$. None of the other control-related ROIs showed an effect. The group difference appeared to be caused mostly by the learned Swahili words (see Figure 4), for which monolinguals showed more activity in the precuneus ([-2 -64 40], $\left.\mathrm{k}=25, p_{S V C}=.016\right)$, left MFG $([-144648], \mathrm{k}=32$, 
$\left.p_{S V C}=.037\right)$, left MTG $\left([-62-36-6], \mathrm{k}=8, p_{S V C}=.031\right)$, and a trend in the left AG $([-50-64$

$\left.34], \mathrm{k}=12, p_{S V C}=.054\right)$. No suprathreshold voxels for the group contrast were observed in the existing and untrained conditions.

Bilinguals showed more overall activation than monolinguals on the whole brain level in the pars opercularis of the right IFG extending into the precentral gyrus, right fusiform gyrus, left middle occipital lobe and left calcarine sulcus. Analysis of the control-related ROIs showed enhanced activation in the left pallidum ([-20 -6 -4], $\left.\mathrm{k}=25, p_{S V C}=.034\right)$. Again, the overall group difference was mainly found in response to the learned Swahili words (see Figure 4): this condition showed a significant cluster in the bilateral middle occipital lobe on the whole brain level and an effect in the left pallidum in the control-related ROI analysis $\left([-20-6-4], \mathrm{k}=22, p_{S V C}=.037\right)$. No effect was found in any of the lexicality ROIs. Additionally, bilinguals showed more activation than monolinguals to existing English words in the left orbital MFG lexicality ROI ([-38 $\left.50-4], \mathrm{k}=21, p_{S V C}=.007\right)$. No interaction was observed between Group and Day (recent/remote).

$<$ Insert Figure 4 about here $>$

\section{Discussion}

The current study investigated whether bilingual experience alters neural mechanisms associated with learning novel words. More specifically, this study tested the hypothesis that bilinguals' experience with learning and using a second language may alter the integration of novel lexical-semantic information with prior knowledge, which may account for the bilingual advantage in word learning that has often been reported in behavioural studies (e.g. Kaushanskaya \& Marian, 2009a; 2009b; Kaushanskya, 2012; Papagno \& Vallar, 1995; Van Hell \& Candia Mahn, 1997; Yoshida et al., 2011; but see Bradley et al., 2013). In the 
Introduction, we discussed three explanations that have been proposed in this literature: bilingual experience may increase sensitivity to semantic information associated with novel words, it may enhance phonological abilities, or it may affect the involvement of cognitive control. English monolinguals and early Spanish-English bilinguals were trained on two sets of Swahili-English word pairs, one set on each of two consecutive days. After training on the second day, participants performed a retrieval task in the fMRI scanner followed by several behavioural memory tests. A primed lexical decision task in which Swahili words primed semantically related English target words was conducted to probe novel words' semantic integration.

The present study showed that bilinguals did not perform better than monolinguals on explicit recall or recognition of novel words, either during training, immediately after training, or after a 24-hour delay. Consistent with previous word learning studies reporting findings of delayed semantic integration (e.g., Bakker et al., 2015; Liu \& Van Hell, 2020; Tamminen \& Gaskell, 2013), a small priming effect from learned Swahili words to semantically related English words was observed for the remote set, learned 24 hours before testing, but not for the recent set, learned just before testing. This pattern is thought to reflect the contribution of an offline integration process, which allows novel words to acquire lexical representations that can form stable neocortical connections with existing words, in line with the Complementary Systems account of word learning (Davis \& Gaskell, 2009). While this effect only reached significance in the monolingual group, we did not observe a Group interaction. So, the behavioural data do not provide support for the hypothesis that bilingual experience leads to faster or stronger lexical integration in bilinguals than in monolinguals, as measured by novel words' ability to prime semantically related existing words. 
Before discussing the fMRI data and observed differences in the neural structures recruited by monolinguals and bilinguals in more detail, we first outline possible explanations for the absence of group differences in the behavioural data.

First, it could be argued that our extended training procedure obscured any bilingual advantage on novel word recall. However, while recognition performance was at ceiling, average scores on the Swahili-cued and English-cued recall tasks ranged between 30\% and $66 \%$ with large standard deviations, suggesting that there was in principle enough variation to detect group differences. Furthermore, there was no group difference in any of the training blocks, which would be expected if the bilingual advantage contributes mainly to early learning effects or fast-mapping (i.e., the initial link made between a novel word and its referent; Kan, Sadagopan, Janich, \& Andrade, 2014). Therefore, the explanation that the extended training procedure obscured differences in the behavioural data of the bilingual and monolingual groups does not seem very likely.

A second explanation for the absence of a group difference on the recall and recognition of novel words, and no semantic priming effect in the bilinguals, relates to the fact that novel words have more orthographic neighbours in the bilinguals' two lexicons combined than in a monolinguals' single lexicon. Note that none of the Swahili words were cognates of English or Spanish words to ensure that lexical overlap of the Swahili words with known languages would not differentially impact novel word learning and consolidation in bilinguals versus monolinguals. Our bilinguals were highly proficient, balanced bilinguals (their BNT scores in English and Spanish did not differ), which implies that the novel Swahili words will have orthographic neighbours in both their English and Spanish mental lexicons. Even though the Swahili words' orthographic neighbourhood density with English and Spanish was fairly low (mean density for the remote, recent, and untrained lists ranged from 0.83-1.80 for English and from 0.90-2.33 for Spanish; Clearpond database, Marian, 
Bartolotti, Chabal, \& Shook, 2012), the summed neighbourhood density is approximately twice as high in bilingual as in monolingual learners. A higher number of orthographic neighbours possibly causes more interference and less stable lexical-semantic representations for newly learned words, yielding a smaller (and nonsignificant) semantic priming effect in bilinguals relative to monolinguals.

A third potential explanation for the absence of group differences in the behavioural measures is that the current paradigm involved only visual exposure to the novel words, due to the restrictions on auditory tasks in the scanner, whereas previous behavioural work reporting a bilingual advantage in word learning has used auditory or auditory-visual paradigms (Kaushanskaya, 2012; Kaushanskaya \& Marian 2009a; 2009b; Papagno \& Vallar, 1995; Van Hell \& Candia Mahn, 1997; Yoshida et al., 2011). In line with this explanation, Bradley et al. (2013) report equal accuracy in monolinguals and bilinguals on a behavioural semantic decision task with newly learned words which had been acquired visually. This pattern raises the possibility that although phonological awareness and memory may not explain the bilingual advantage entirely (Kaushanskaya, 2012; Kaushanskaya \& Marian 2009a), behavioural observations of word learning differences related to bilingual experience may be restricted to spoken word learning or combined auditory-visual word learning.

We now discuss the neural findings, and the observed differences in neural structures recruited by monolinguals and bilinguals. As we will see, these data indicate that monolinguals and bilinguals recruit overlapping but non-identical neural systems to acquire novel words, in line with the proposal put forward by Bradley et al. (2013) that monolinguals and bilinguals employ different strategies in word learning that rely on distinct neural mechanisms. Bradley et al. report increased activation for monolinguals in areas related to cognitive control, in the right inferior/middle frontal gyri, an effect that was replicated in our data. This pattern was interpreted as a signature of more controlled response selection and 
competitor inhibition in monolinguals, following Abutalebi and Green (2007). Our observation of stronger IFG activation in monolinguals compared to bilinguals is consistent with this view and, combined with the finding that bilinguals showed smaller mixing costs than monolinguals on the Flanker task - taken to reflect bilinguals' experiences in monitoring and coordinating two languages (e.g., Barac \& Bialystok, 2012; Gold et al., 2013; Yang et al., 2016; Wiseheart et al., 2016) - suggests that the bilinguals' response system is more flexible and better equipped to monitor and coordinate multiple streams of information without so much conscious effort. The bilingual experience of learning and using multiple languages may thus change the recruitment of brain regions in novel word learning, analogous to the recently proposed bilingual anterior-to-posterior and subcortical shift (BAPSS) associated with nonlinguistic cognitive and executive function tasks (Grundy, Anderson, \& Bialystok, 2017). This shift takes the form of less recruitment of frontal and executive regions and greater recruitment of posterior/subcortical regions by bilinguals relative to monolinguals. In line with the BAPSS proposal, our data also seem to suggest that bilinguals recruit less frontal regions than monolinguals and more recruitment of the basal ganglia region. Based on their extensive review of the literature, Grundy et al. further argue that this pattern reflects efficiency rather than functional dedifferentiation, because studies have observed different neural patterns between bilinguals and monolinguals, but no behavioural differences (as did the present study).

Furthermore, the bilinguals in Bradley et al.'s study exhibited more activation in the left putamen, as was also observed in the current data. (Note that while Bradley et al. label their cluster as putamen, its peak voxel is just within the AAL-defined pallidum on the pallidum/putamen border. The peak voxel in our data is slightly more medial in the pallidum.) They argued that this pattern reflects bilinguals' greater reliance on articulatory motor processes in mapping novel and existing words, given the known involvement of the 
putamen/pallidum in motor processes and control of articulatory processes (e.g., Wildgruber, Ackermann, \& Grodd, 2001; Wise, Green, Buchel, \& Scott, 1999; for review, see ViñasGuasch \& Wu, 2017), and the finding of increased grey matter density in the left putamen for multilinguals relative to monolinguals (Abutalebi et al., 2013; see also Burgaleta, Sanjuán, Ventura-Campos, Sebastián-Gallás, \& Ávila, 2016). If bilinguals’ long-term experience navigating two languages and controlling articulatory processes in two languages induced structural changes in the putamen and has prepared bilinguals to rely on articulatory motor processing when integrating novel words into their existing mental lexicon, then this may also explain why a bilingual advantage in word learning is prevalent in behavioural studies employing auditory or combined auditory-visual word learning strategies, and why studies using visual-only learning strategies (the present study and Bradley et al., 2013) did not observe a bilingual advantage. A direct, within-subjects comparison of auditory and visual word learning in monolinguals and bilinguals is necessary to establish whether bilingual experience enhances both auditory and visual word learning, or whether experience-induced changes are restricted to auditory word learning.

Several regions that responded selectively to existing words, as compared to untrained Swahili words (i.e., pseudowords), also showed enhanced responses to learned Swahili words in monolinguals versus bilinguals. This network included the left precuneus, middle frontal gyrus (MFG) and middle temporal gyrus (MTG), and less reliably the left angular gyrus (AG). Previous work indicates that the left MTG in particular is important for the lexicalisation and semantic integration of novel words (e.g., Bakker-Marshall et al., 2018; Takashima et al., 2014), in line with the idea that this region houses lexical representations that bind word-form information to distributed semantic knowledge (Gow, 2012; Hickok \& Poeppel, 2004; 2007; Lau, Phillips \& Poeppel, 2008). The increased activation in areas sensitive to lexicality in monolinguals, especially the left MTG, may therefore reflect 
stronger lexical representations of newly learned words, possibly underlying the differences in behavioural priming across groups.

One potential explanation for this difference in lexical activation patterns lies in the difference in English vocabulary size between the monolingual and bilingual groups. The bilinguals scored lower on the English naming task than monolinguals, consistent with a large-sample comparison of monolingual and bilingual adults on a similar standardised picture-naming task (Bialystok \& Luk, 2012). Their smaller English vocabulary size may have impaired bilinguals' ability to encode and integrate the Swahili words with their English translations, either through increased task demands or because fewer representations were available for novel words to form connections with, thus counteracting any positive effects of bilingualism on word learning (including the notion that bilingual experience may increase sensitivity to semantic information associated with novel words (e.g., Kaushanskaya and Rechtzigel, 2012), as discussed in the Introduction). However, we did not find a correlation between bilinguals' English naming scores and any of the explicit novel word memory measures (all $p>.12$ ) or the priming effect for remote words $(p=.877$ ), although some caution is warranted in interpreting these findings since these correlation analyses may be underpowered. Furthermore, the enhanced activation in the MTG for monolinguals versus bilinguals remained significant when controlling for naming performance $\left(p_{F W E}=.04\right)$, although the precuneus and MFG effects no longer reached significance (possibly due to a loss of power, as naming data was not available for two participants).

An alternative, and tentative, explanation we propose here is that novel words may be incorporated in the monolingual and bilingual lexicons in fundamentally different ways. One of the main computational arguments for offline consolidation is that temporarily encoding novel information separately from prior knowledge circumvents the problem of catastrophic interference: the erasing of existing memories in a distributed connectionist network by the 
sudden introduction of new information (McClelland et al., 1995; McClosky \& Cohen, 1989). If the neural networks supporting lexical-semantic representations overlap across a bilingual's languages, the denser bilingual lexicon may be more vulnerable to catastrophic interference than the monolingual lexicon. To overcome this problem, bilinguals more so than monolinguals may need to prioritise slower and more gradual interleaving of novel and existing words into the neocortical network, and rely more strongly and possibly for a longer period of time on hippocampal encoding of novel words. Given the faster decay of hippocampal information (see Frankland \& Bontempi, 2005 for a discussion of possible mechanisms), this is consistent with the finding in prior behavioural work that the bilingual advantage observed immediately after learning dissipates over time (Kaushanskaya \& Marian, 2009a; 2009b; Kaushanskaya, 2012).

Thus, as observed in behavioural work (particularly in studies using auditory or combined auditory-visual word learning strategies), bilinguals may generally have an advantage on immediate explicit memory tasks for novel words (e.g. Kaushanskaya \& Marian, 2009a; 2009b; Kaushanskya, 2012; Papagno \& Vallar, 1995; Van Hell \& Candia Mahn, 1997; Yoshida et al., 2011), which can be performed based on an episodic, hippocampally bound memory trace. Monolinguals on the other hand may integrate novel words more rapidly into the neocortical lexicon bound by the left MTG, which in the current paradigm could have enabled novel words to interact with existing words in the semantic priming task (which only reached significance in the monolingual group). Our data did not reveal increased hippocampal activation for bilinguals, but hippocampal effects are not always readily observed and straightforward to interpret during word retrieval (e.g. Davis, DiBetta, MacDonald, \& Gaskell, 2009; Takashima et al., 2014). Future work employing paradigms optimised to test this hypothesis, perhaps investigating hippocampal activity during associative encoding rather than retrieval, may start to address this issue. 
In conclusion, the current study indicates that monolinguals and bilinguals reach largely comparable behavioural performance levels in novel word learning, but do so by recruiting partially overlapping but non-identical neural systems to acquire novel words. Depending on which features of novel word knowledge are emphasised by a particular experimental paradigm, bilingual experience may induce both advantages and disadvantages in novel word learning. 


\section{References}

Abutalebi, J. \& Green, D. (2007). Bilingual language production: The neurocognition of language representation and control. Journal of Neurolinguistics, 20, 242-275.

Abutalebi, J. \& Green, D. W. (2016). Neuroimaging of language control in bilinguals: neural adaptation and reserve. Bilingualism: Language and Cognition, 19 (4), 689-698.

Abutalebi, J., Rosa, P. A. D., Castro Gonzaga, A. K., Keim, R., Costa, A., \& Perani, D. (2013). The role of the left putamen in multilingual language production. Brain and Language, 125, 307-315.

Baayen, R. H., Piepenbrock, R., \& Gulikers, L. (1995). The CELEX lexical database [webcelex]. Philadelphia, PA: University of Pennsylvania Linguistic Data Consortium.

Bakker, I., Takashima, A., Van Hell, J. G., Janzen, G., \& McQueen, J. M. (2014). Competition from unseen or unheard novel words: Lexical consolidation across modalities. Journal of Memory and Language, 73, 116-130.

Bakker, I., Takashima, A., Van Hell, J. G., Janzen, G., \& McQueen, J. M. (2015). Tracking lexical consolidation with ERPs: Lexical and semantic priming effects on N400 and LPC responses to newly-learned words. Neuropsychologia, 79, 33-41.

Bakker-Marshall, I., Takashima, A., Schoffelen, J-M., Van Hell, J. G., Janzen, G., \& McQueen, J. M. (2018). Theta-band oscillations in the middle temporal gyrus reflect novel word consolidation. Journal of Cognitive Neuroscience, 30, 621-633.

Barac, R., \& Bialystok, E. (2012). Bilingual effects on linguistic and cognitive development: role of language, cultural background, and education. Child Development, 83, 413-422.

Bartolotti, J., \& Marian, V. (2012). Language learning and control in monolinguals and bilinguals. Cognitive Science, 36, 1129-1147.

Bartolotti, J., Marian, V., Schroeder, S. R., \& Shook, A. (2011). Bilingualism and inhibitory control influence statistical learning of novel word forms. Frontiers in Psychology, 1-10. 
Bavelier, D. \& Davidson, R. J. (2013). Brain training: Games to do you good. Nature, 494, 425-426.

Bialystok, E. (2017). The bilingual adaptation: How minds accommodate experience. Psychological Bulletin, 143, 233-262.

Bialystok, E., Craik, F. I. M., \& Luk, G. (2012). Bilingualism: Consequences for mind and brain. Trends in Cognitive Sciences, 16, 240-50.

Bialystok, E., \& Luk, G. (2012). Receptive vocabulary differences in monolingual and bilingual adults. Bilingualism: Language and Cognition, 15, 397-401.

Bialystok, E., Luk, G., \& Kwan, E. (2005). Bilingualism, biliteracy, and learning to read: Interactions among languages and writing systems. Scientific Studies of Reading, 9, 4361.

Bialystok, E., Majumder, S., \& Martin, M. M. (2003). Developing phonological awareness: Is there a bilingual advantage? Applied Psycholinguistics, 24, 27-44.

Bradley, K. a L., King, K. E., \& Hernandez, A. E. (2013). Language experience differentiates prefrontal and subcortical activation of the cognitive control network in novel word learning. NeuroImage, 67, 101-110.

Burgaleta, M., Sanjuán, A., Ventura-Campos, N., Sebastián-Gallás, N., \& Ávila, C. (2016). Bilingualism at the core of the brain. Structural differences between bilinguals and monolinguals revealed by subcortical shape analysis. NeuroImage, 125, 437-445.

Cenoz, J., \& Valencia, J. F. (1994). Additive trilingualism: Evidence from the Basque Country. Applied Psycholinguistics, 15, 195-207.

Costa, A., Hernández, M., \& Sebastián-Gallés, N. (2008). Bilingualism aids conflict resolution: Evidence from the ANT task. Cognition, 106, 59-86.

Davis, M. H., Di Betta, A. M., McDonald, M. J., \& Gaskell, M. G. (2009). Learning and consolidation of novel spoken words. Journal of Cognitive Neuroscience, 21, 803-820. 
Davis, M. H., \& Gaskell, M. G. (2009). A Complementary Systems account of word learning: Neural and behavioural evidence. Philosophical Transactions of the Royal Society of London. Series B, Biological Sciences, 364, 3773-3800.

De Groot, A. M. (1989). Representational aspects of word imageability and word frequency as assessed through word association. Journal of Experimental Psychology: Learning, Memory, and Cognition, 15, 824-845.

De Groot, A. M. B. (1992). Bilingual lexical representation: A closer look at conceptual representations. In R. Frost \& L. Katz (Eds.), Orthography, phonology, morphology, and meaning (pp. 389-412). Amsterdam: North-Holland.

Draganski, B., Gaser, C., Busch, V., Schuierer, G., Bogdahn, U., \& May, A. (2004). Nature, $427,311-313$.

Dumay, N., \& Gaskell, M. G. (2007). Sleep-associated changes in the mental representation of spoken words. Psychological Science, 18, 35-39.

Dumay, N., \& Gaskell, G. M. (2012). Overnight lexical consolidation revealed by speech segmentation. Cognition, 123, 119-132.

Eriksen, B. A., \& Eriksen, C. W. (1974). Effects of noise letters upon the identification of a target letter in a nonsearch task. Perception \& Psychophysics, 16, 143-149.

Frankland, P. W., \& Bontempi, B. (2005). The organization of recent and remote memories. Nature Reviews Neuroscience, 6, 119-130.

Gaskell, M. G., \& Dumay, N. (2003). Lexical competition and the acquisition of novel words. Cognition, 89, 105-132.

Gold, B. T., Kim, C., Johnson, N. F., Kryscio, R. J., \& Smith, C. D. (2013). Lifelong bilingualism maintains neural efficiency for cognitive control in aging. Journal of Neuroscience, 33, 397-396. 
Gow, D. W. (2012). The cortical organization of lexical knowledge: A dual lexicon model of spoken language processing. Brain and Language, 121, 273-288.

Grundy, J. G., Anderson, J. A. E., \& Bialystok, E. (2017). Neural correlates of cognitive processing in monolinguals and bilinguals. Annals of the New York Academy of Sciences, 1396, 183-201.

Herholz, S. C., \& Zatorre, R. J. (2012). Musical training as framework for brain plasticity: behavior, function, and structure. Neuron, 76, 486-502.

Hickok, G., \& Poeppel, D. (2004). Dorsal and ventral streams: A framework for understanding aspects of the functional anatomy of language. Cognition, 92, 67-99.

Hickok, G., \& Poeppel, D. (2007). The cortical organization of speech processing. Nature Reviews Neuroscience, 8, 393-402.

Hilchey, M. D., \& Klein, R. M. (2011). Are there bilingual advantages on nonlinguistic interference tasks? Implications for the plasticity of executive control processes. Psychonomic Bulletin \& Review, 18, 625-658.

Hirosh, Z., \& Degani, T. (2018). Direct and indirect effects of multilingualism on novel word learning: An integrative review. Psychonomic Bulletin \& Review, 25, 892-916.

Jin, Y.-S. (1990). Effects of concreteness on cross-language priming in lexical decisions. Perceptual and Motor Skills, 70, 1139-1154.

Kan, P., Sadagopan, N., Janich, L., \& Andrade, M. (2014). The effects of speech practice on fast mapping in monolingual and bilingual speakers. Journal of Speech, Language, and Hearing Research, 57, 929-941.

Kaushanskaya, M. (2012). Cognitive mechanisms of word learning in bilingual and monolingual adults: The role of phonological memory. Bilingualism: Language and Cognition, 15, 470-489. 
Kaushanskaya, M., \& Marian, V. (2009a). The bilingual advantage in novel word learning. Psychonomic Bulletin \& Review, 16, 705-10.

Kaushanskaya, M., \& Marian, V. (2009b). Bilingualism reduces native-language interference during novel-word learning. Journal of Experimental Psychology. Learning, Memory, and Cognition, 35, 829-35.

Kaushanskaya, M., \& Rechtzigel, K. (2012). Concreteness effects in bilingual and monolingual word learning. Psychonomic Bulletin \& Review, 19, 935-941.

Kovelman, I., Baker, S. A., \& Petitto, L. A. (2008). Bilingual and monolingual brains compared: a functional magnetic resonance imaging investigation of syntactic processing an a possible "neural signature" of bilingualism. Journal of Cognitive Neuroscience, 20, 153-169.

Lau, E. F., Phillips, C., \& Poeppel, D. (2008). A cortical network for semantics: (de)constructing the N400. Nature Reviews Neuroscience, 9, 920-933.

Leach, L., \& Samuel, A. G. (2007). Lexical configuration and lexical engagement: When adults learn new words. Cognitive Psychology, 55, 306-353.

Lehtonen, M., Soveri, A., Laine, A., Järvenpää, J., de Bruin, A. \& Antfolk, J. (2018). Is bilingualism associated with enhanced executive functioning in adults? A meta-analytic review. Psychological Bulletin, 144(4), 394-425.

Li, P., Legault, J., \& Litcofsky, K. A. (2014). Neuroplasticity as a function of second language learning: Anatomical changes in the human brain. Cortex, 58, 301-324.

Lindsey, S., \& Gaskell, M. G. (2013). Lexical integration of novel words without sleep. Journal of Experimental Psychology: Learning, Memory, and Cognition, 39, 608-622.

Liu, Y., \& Van Hell, J. G. (2020). Learning novel word meanings: an ERP study on lexical consolidation in monolingual, inexperienced foreign language learners. Language Learning, 70: S2, 45-74. 
Luk, G. (2008). The anatomy of the bilingual influence on cognition: Levels of functional use and proficiency of language. $\mathrm{PhD}$ dissertation, York University Toronto.

Maguire, E. A., Gadian, D. G., Johnsrude, I. S., Good, C. D., Ashburner, J., Frackowiak, R. S. \& Frith, C. D. (2000). Navigation-related structural changes in the hippocampi of taxi drivers. Proceedings of the National Academy of the United States of America, 97, 43984403.

Marian, V., Bartolotti, J., Chabal, S., \& Shook, A. (2012). CLEARPOND: Cross-Linguistic Easy-Access Resource for Phonological and Orthographic Neighborhood Density. PLoS ONE, 7(8): e43230

Marian, V., \& Spivey, M. (2003). Competing activation in bilingual language processing: Within- and between-language competition. Bilingualism: Language and Cognition, 6, 97-115.

Marr, D. (1970). A theory for cerebral neocortex. Proceedings of the Royal Society Series B, $176,161-234$.

McClelland, J. L., McNaughton, B. L., \& O’Reilly, R. C. (1995). Why there are complementary learning systems in the hippocampus and neocortex: Insights from the successes and failures of connectionist models of learning and memory. Psychological Review, 102, 419-457.

McClosky, M., \& Cohen, N. J. (1989). Catastrophic interference in connectionist networks: The sequential learning problem. In G. H. Bower (Ed.), The psychology of learning and motivation (pp. 109-165). New York: Academic Press.

Paap, K. R., Johnson, H. A., \& Sawi, O. (2015). Bilingual advantages in executive functioning either do not exist or are restricted to very specific and undetermined circumstances. Cortex, 69, 265-278. 
Papagno, C., \& Vallar, G. (1995). Verbal short-term memory and vocabulary learning in polyglots. The Quarterly Journal of Experimental Psychology. A, Human Experimental Psychology, 48, 98-107.

Paivio, A., Walsh, M., \& Bons, T. (1994). Concreteness effects on memory: When and why? Journal of Experimental Psychology. Learning, Memory, and Cognition, 20, 1196-1204.

Pliatsikas, C. (2020). Understanding structural plasticity in the bilingual brain: The Dynamic Restructuring Model. Bilingualism: Language and Cognition, 23, 459-471.

Sanz, C. (2000). Bilingual education enhances third language acquisition: Evidence from Catalonia. Applied Psycholinguistics, 21, 23-44.

Squire, L. R., \& Alvarez, P. (1995). Retrograde amnesia and memory consolidation: A neurobiological perspective. Current Opinion in Neurobiology, 5, 169-177.

Stocco, A., Yamasaki, B., Natalenko, R., \& Prat, C. S. (2014). Bilingual brain training: A neurobiological framework of how bilingual experience improves executive function. International Journal of Bilingualism, 18(1), 67-92.

Takashima, A., Bakker, I., van Hell, J. G., Janzen, G., \& McQueen, J. M. (2014). Richness of information about novel words influences how episodic and semantic memory networks interact during lexicalization. NeuroImage, 84, 265-278.

Takashima, A., Bakker-Marshall, I., Van Hell, J. G., McQueen, J. M., \& Janzen, G. (2019). Neural correlates of word learning in children. Developmental Cognitive Neuroscience, 37,100649 .

Tamminen, J., \& Gaskell, M. G. (2013). Novel word integration in the mental lexicon: Evidence from unmasked and masked semantic priming. Quarterly Journal of Experimental Psychology, 66, 1001-1025.

Tse, D., Langston, R. F., Kakeyama, M., Bethus, I., Spooner, P. A., Wood, E. R., ... Morris, R. G. M. (2007). Schemas and memory consolidation. Science, 316, 76-82. 
Tzourio-Mazoyer, N., Landeau, B., Papathanassiou, D., Crivello, F., Etard, O., Delcroix, N., ... Joliot, M. (2002). Automated anatomical labeling of activations in SPM using a macroscopic anatomical parcellation of the MNI MRI single-subject brain. NeuroImage, $15,273-289$.

Van der Ven, F., Takashima, A., Segers, E., \& Verhoeven, L. (2015). Learning word meanings: Overnight integration and study modality effects. PLoS ONE, 10(5), e0124926.

Van Hell, J. G., \& Candia Mahn, A. (1997). Keyword mnemonics versus rote rehearsal: Learning concrete and abstract foreign words by experienced and inexperienced learners. Language Learning, 47, 507-546.

Van Hell, J. G., \& De Groot, A. M. B. (1998a). Disentangling context availability and concreteness in lexical decision and word translation. The Quarterly Journal of Experimental Psychology Section A, 51, 41-63.

Van Hell, J. G., \& De Groot, A. M. B. (1998b). Conceptual representation in bilingual memory: Effects of concreteness and cognate status in word association. Bilingualism: Language and Cognition, 1(3), 193-211.

Van Heuven, W. J. B., Dijkstra, T., \& Grainger, J. (1998). Orthographic neighborhood effects in bilingual word recognition. Journal of Memory and Language, 39, 458-483.

Viñas-Guasch, N., \& Wu, Y. J. (2017). The role of the putamen in language: a meta-analytic connectivity modeling study. Brain Structure and Function, 222, 3391-4004.

Wildgruber, D., Ackermann, H., \& Grodd, W. (2001). Differential contributions of motor cortex, basal ganglia, and cerebellum to speech motor control: Effects of syllable repetition rate evaluated by fMRI. NeuroImage, 13, 101-109.

Wise, R. J. S., Greene, J., Buchel, C., \& Scott, S. K. (1999). Brain regions involved in articulation. The Lancet, 353, 1057-1061. 
Wiseheart, M., Viswanathan, M., Bialystok, E. (2016). Flexibility in task switching by monolinguals and bilinguals. Bilingualism: Language and Cognition, 19, 141-146.

Yang, H., Hartanto, A, \& Yang, S. (2016). The complex nature of bilinguals' language use modulates task-switching outcomes. Frontiers in Psychology, 7, 560.

Yoshida, H., Tran, D. N., Benitez, V., \& Kuwabara, M. (2011). Inhibition and adjective learning in bilingual and monolingual children. Frontiers in Psychology, 2, 1-14. 


\section{Footnotes}

${ }^{1}$ This claim is supported by data showing that, relative to abstract words, bilinguals are faster to translate concrete words (e.g. Van Hell \& De Groot, 1998a), show stronger cross-language priming (Jin, 1990), and provide more similar word association responses across their two languages in response to concrete words (Van Hell \& De Groot, 1998b). 
Table 1. Novel, untrained and existing stimuli sets. List A and B were counterbalanced across subjects as either the recent or remote condition.

\begin{tabular}{|c|c|c|c|c|c|}
\hline $\begin{array}{l}\text { List A } \\
\text { Swahili }\end{array}$ & $\begin{array}{l}\text { English } \\
\text { Translation }\end{array}$ & $\begin{array}{l}\text { List B } \\
\text { Swahili }\end{array}$ & $\begin{array}{l}\text { English } \\
\text { Translation }\end{array}$ & $\begin{array}{l}\text { Untrained } \\
\text { Swahili }\end{array}$ & $\begin{array}{l}\text { Existing } \\
\text { English }\end{array}$ \\
\hline bata & duck & bongo & brain & barafu & barn \\
\hline dubu & bear & buibui & spider & divai & belt \\
\hline funza & worm & bundi & owl & gari & cake \\
\hline gombe & cow & chanda & finger & ghuba & deer \\
\hline goti & knee & chura & frog & hatari & doll \\
\hline hema & tent & dawati & desk & jicho & flag \\
\hline jiji & city & duara & wheel & kesho & leek \\
\hline joka & snake & fagio & broom & kivuli & lion \\
\hline kalamu & pen & farasi & horse & kondoo & moon \\
\hline kioo & mirror & fupa & bone & kopo & nose \\
\hline kitabu & book & hori & canoe & kubwa & arrow \\
\hline kiti & chair & jabali & mountain & marefu & basket \\
\hline kofia & hat & jani & leaf & mashua & camel \\
\hline lango & door & kanzu & coat & mayai & candle \\
\hline maji & water & kawe & rock & mbuzi & hamster \\
\hline meli & ship & kiatu & shoe & mguu & helmet \\
\hline ndege & airplane & kijiko & spoon & moshi & island \\
\hline nyuki & bee & kisu & knife & mpira & lemon \\
\hline nyundo & hammer & kitanda & bed & mshipi & lizard \\
\hline panya & mouse & mavazi & dress & ndio & lorry \\
\hline pete & ring & mfuko & bag & sana & motor \\
\hline pwani & beach & mofa & bread & sasa & onion \\
\hline ramani & map & paka & cat & shavu & otter \\
\hline sila & bucket & pombe & beer & sungura & piano \\
\hline tondo & snail & samaki & fish & tisa & porch \\
\hline viazi & potato & siafu & ant & twiga & skate \\
\hline waridi & rose & tariki & road & ubao & sweat \\
\hline weni & plant & tembo & elephant & usubi & tongue \\
\hline wingu & cloud & tofaa & apple & watu & whale \\
\hline zulia & rug & uzio & fence & zizi & zipper \\
\hline
\end{tabular}



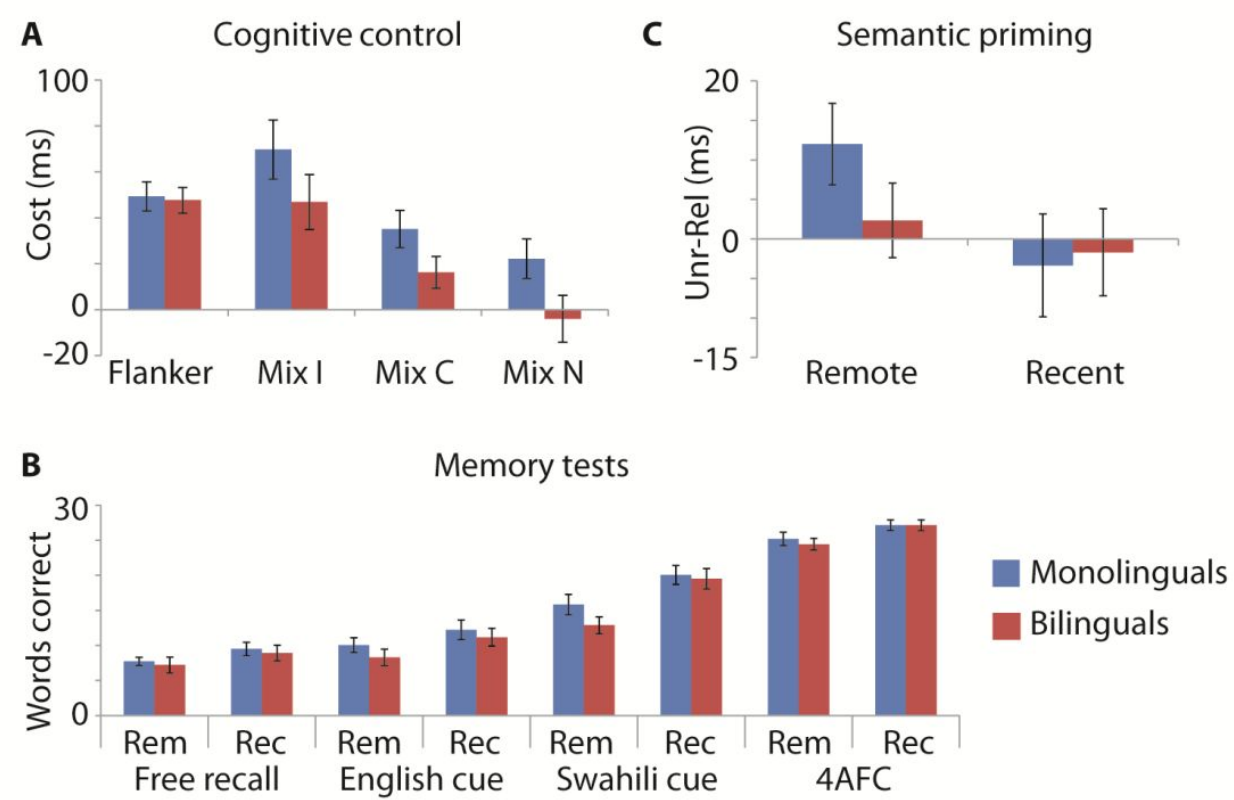

Figure 1. Behavioural results. A: Left-most bars indicate the Flanker effect (congruent incongruent trials in the blocked conditions) for monolinguals (blue) and bilinguals (red). The next three bar clusters indicate the mixing cost (trials in the mixed block relative to the same trial type in the blocked conditions) for incongruent (I), congruent (C), and neutral (N) trials. B: Memory performance for recently learned (Rec) and remote (Rem) words on the Free recall task, typing the Swahili word in response to an English cue, typing the English word in response to a Swahili cue, and 4AFC Swahili-to-English matching. The total number of words learned on each day was 30. C: Semantic priming effects (unrelated - related primetarget pairs) for the remote and recently learned words. Error bars denote standard error of the mean.

$127 \times 85 \mathrm{~mm}(300 \times 300 \mathrm{DPI})$ 


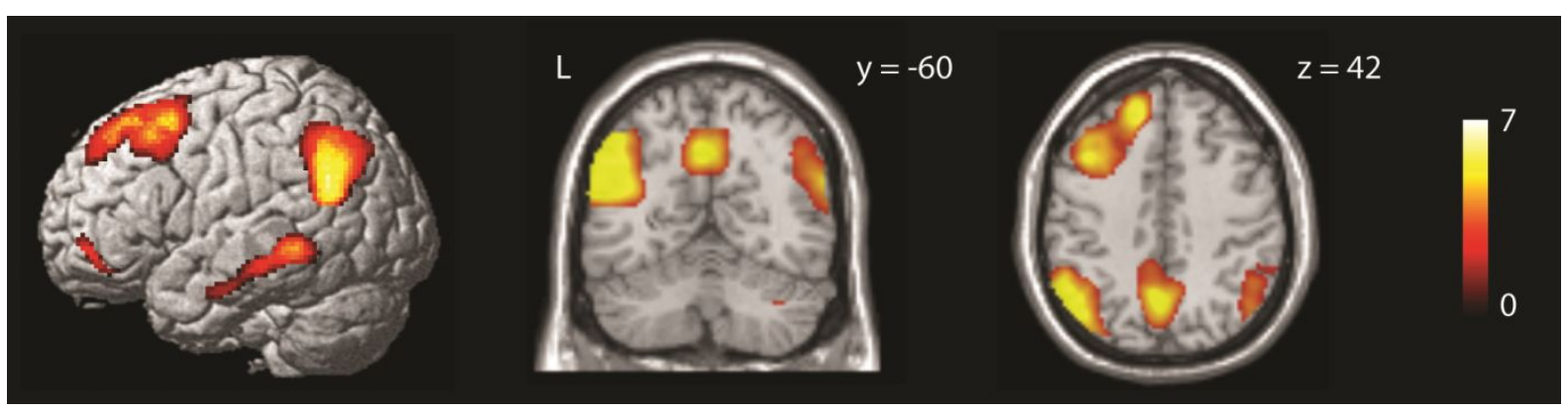

Figure 2. Greater activation for existing English words than untrained Swahili words (lexicality effect), across both groups. Thresholded at voxel $p_{F W E}=.05$.

$60 \times 39 \mathrm{~mm}(300 \times 300 \mathrm{DPI})$ 


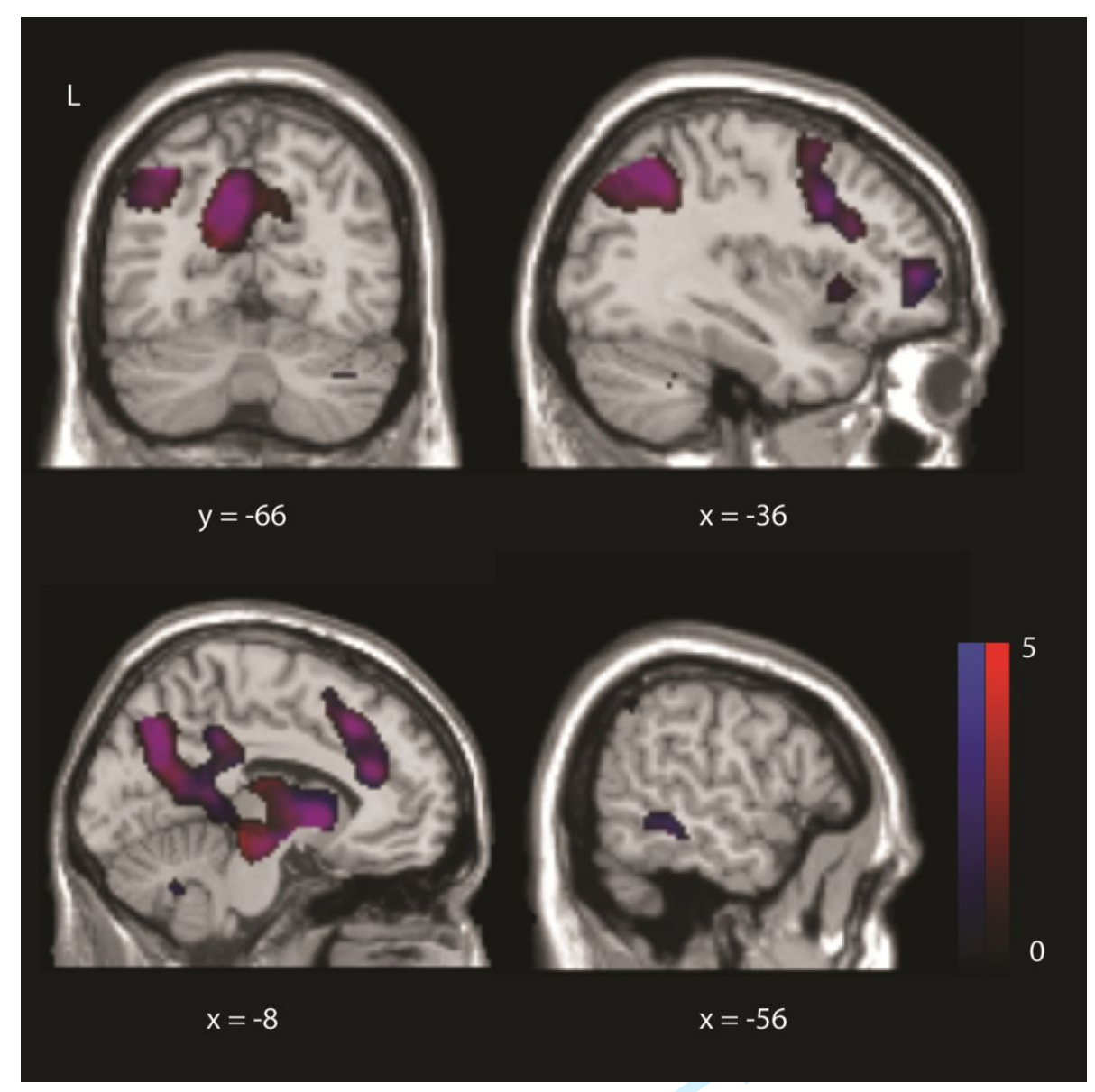

Figure 3. Enhanced activation for recent (blue) and remote (red) words (overlap in purple) versus untrained Swahili words, across groups. Thresholded at voxel $p_{u n c}=.001$, except for the bottom right panel which depicts the MTG cluster at $p_{u n c}=.01$ for illustration purposes.

$119 \times 119 \mathrm{~mm}(300 \times 300 \mathrm{DPI})$ 


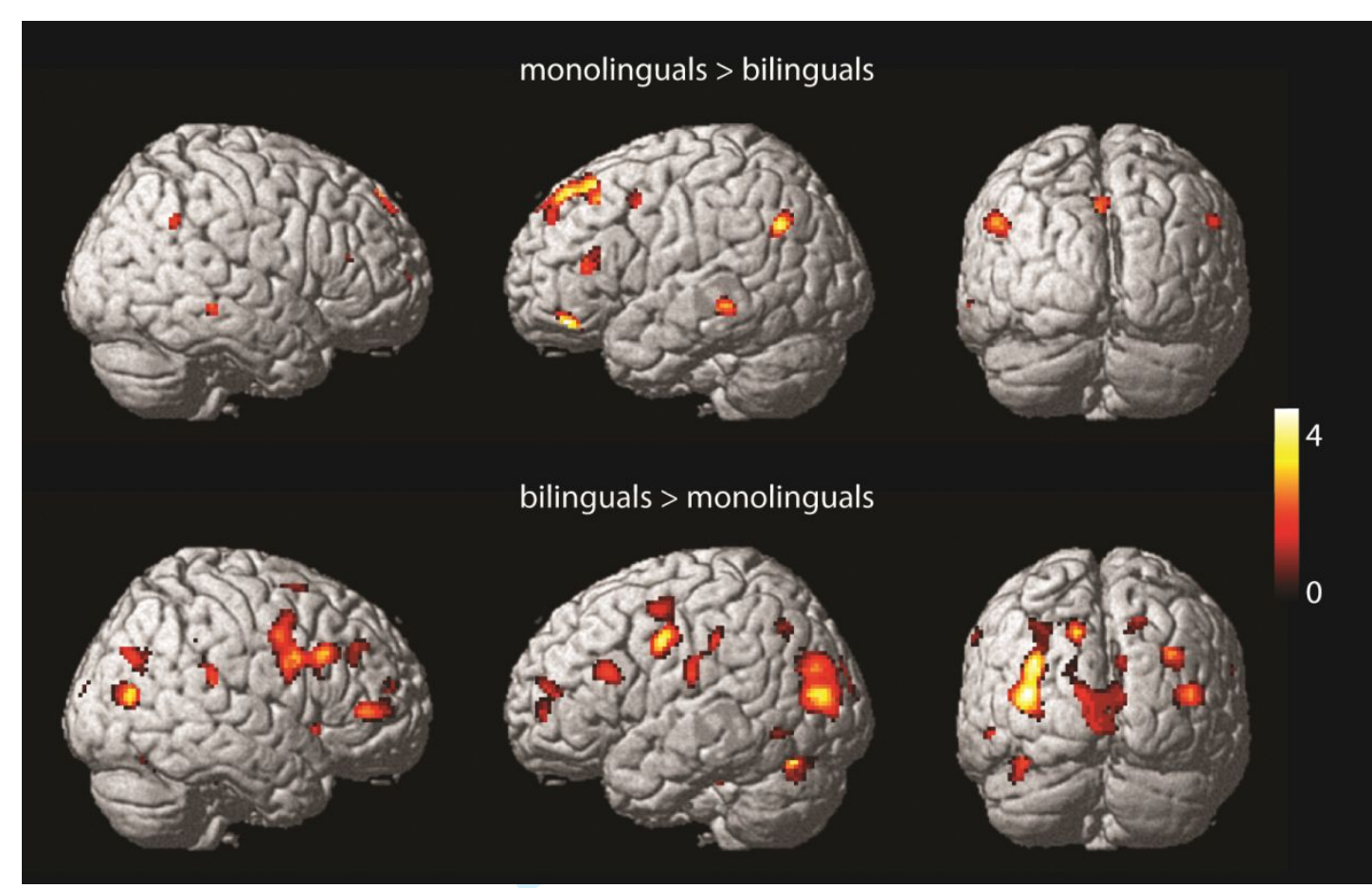

Figure 4. Activation differences between monolinguals and bilinguals in response to learned (recent and remote) Swahili words. Thresholded at voxel $p_{u n c}=.01$ for illustration purposes.

144x92mm (300 x 300 DPI) 
Figure captions.

Figure 1. Behavioural results. A: Left-most bars indicate the Flanker effect (congruent incongruent trials in the blocked conditions) for monolinguals (blue) and bilinguals (red). The next three bar clusters indicate the mixing cost (trials in the mixed block relative to the same trial type in the blocked conditions) for incongruent (I), congruent (C), and neutral (N) trials. B: Memory performance for recently learned (Rec) and remote (Rem) words on the Free recall task, typing the Swahili word in response to an English cue, typing the English word in response to a Swahili cue, and 4AFC Swahili-to-English matching. The total number of words learned on each day was 30. C: Semantic priming effects (unrelated - related primetarget pairs) for the remote and recently learned words. Error bars denote standard error of the mean.

Figure 2. Greater activation for existing English words than untrained Swahili words (lexicality effect), across both groups. Thresholded at voxel $p_{F W E}=.05$.

Figure 3. Enhanced activation for recent (blue) and remote (red) words (overlap in purple) versus untrained Swahili words, across groups. Thresholded at voxel $p_{u n c}=.001$, except for the bottom right panel which depicts the MTG cluster at $p_{u n c}=.01$ for illustration purposes.

Figure 4. Activation differences between monolinguals and bilinguals in response to learned (recent and remote) Swahili words. Thresholded at voxel $p_{u n c}=.01$ for illustration purposes. 


\section{Highlights}

- No bilingual-monolingual behavioural difference on memory for newly learned words

- fMRI data suggest monolinguals rely more strongly on cognitive control networks

- Bilinguals recruit more articulatory motor processes

- Bilingual advantages may depend on word learning modality

- Bilinguals showed smaller mixing costs than monolinguals on Flanker task. 